\title{
On multi-fiber beam model with coupled axial and bending response
}

\author{
Géraldine Casaux-Ginestet* — Adnan Ibrahimbegovic** \\ * Université de Toulouse ; UPS, INSA ; LMDC \\ 135, avenue de Rangueil, F-31077 Toulouse cedex 04 \\ geraldine.casaux-ginestet@insa-toulouse.fr \\ ** Laboratoire de Mécanique et Technologie, F-94235 Cachan cedex \\ adnan.ibrahimbegovic@ens-cachan.fr
}

\begin{abstract}
Being able to reproduce the coupling between normal and flexural components is a crucial point for multifiber beam element modeling. The aim of this work is to propose a solution for this coupling, using the incompatible modes method. Applied to axial interpolation functions, this method makes possible the coupled description of normal and flexural responses with the same order of accuracy. The enrichment is presented in detail then its performances are highlighted thanks to an academic test.

RÉSUMÉ. La capacité à reproduire le couplage effort normal/moment de flexion est un point clef de la modélisation par éléments de poutre multifibre. L'objectif de ce travail est de proposer une solution pour ce couplage en utilisant la méthode des modes incompatibles. Appliquée aux fonctions d'interpolation du déplacement axial, cette méthode permet une description couplée des réponses axiale et de flexion avec le même niveau de précision. L'enrichissement de l'élément est présenté en détails puis ses performances sont mises en évidence grâce à un cas test académique.

KEYWORDS: multifiber beam element, normal and flexural coupling, incompatible modes.

MOTS-CLÉS: élément de poutre multifibre, couplage effort normal/flexion, modes incompatibles.
\end{abstract}

DOI:10.3166/EJCM.20.411-426 ( 2011 Lavoisier Paris 


\section{Introduction}

Predicting the behavior of structures subjected to severe loadings, requires a precise modeling of the structure, even within the framework of simplified methods. Indeed, very important couplings are very often highlighted (Ragueneau et al., 2003). The multifiber beam approach (Spacone et al., 1996) has many advantages: the computing time is reduced and thus makes it possible to treat more cases or to perform parametric studies. Among the highlighted couplings, the normal force bending moment coupling seems a major stake of the multifiber beam element modeling. The simultaneity of a high tension force and a high bending rotation can thus lead to a premature ruin of the structure, which has to be predicted. However, there is no coupling between normal and flexural responses in a basic multifiber beam element, and the axial strain is constant over the element. It is thus necessary to enrich this axial deformation's calculation. In order to simplify the presentation, Euler-Bernoulli beam kinematics is used. An enrichment of the interpolation of the axial deformation using the incompatible modes method (Ibrahimbegovic et al., 1991) is proposed and established in the finite element code FEAP (Taylor, 1996). Then its performances are highlighted thanks to an academic test.

\section{Euler-Bernoulli multifiber beam element}

\subsection{Presentation}

A traditional beam finite element can only describe one element made up of one material. It is thus not adapted for composite structures like reinforced concrete ones. A way of enriching this element is to make a multifiber beam element of it. Using multifiber beam elements has many advantages. Indeed, adopting beam kinematics in cross-sections, they make it possible to treat complex problems with nonlinear constitutive laws within a one-dimensional framework. In the cross section, various fibers are described with various constitutive laws. After integration along the cross-section, there are few degrees of freedom, compared to the complexity of the modeled structure.

The Euler-Bernoulli multifiber beam element is a finite element with two nodes which can be a bi- or three-dimensional element. It is defined by its section geometry and its length. The section's kinematics is the Euler-Bernoulli continuous beam one (Figure 1). Two Gauss points are used for integration. Thus, with kinematics assumptions and nodes displacements, one obtains, thanks to the shape functions, the axial strain and the section curvature, at the Gauss points' locations. The deformation of each fiber and then its normal stress (thanks to one-dimensional constitutive laws) can be calculated. Integration on the section, then on the element, leads to the normal force and bending moment at the nodes. If constitutive laws are elasticity ones, 


$$
\left(\begin{array}{l}
N \\
M
\end{array}\right)=\left(\begin{array}{cc}
\int_{S} E_{P} d S & -\int_{S} E_{P} y d S \\
-\int_{S} E_{P} y d S & \int_{S} E_{P} y^{2} d S
\end{array}\right)\left(\begin{array}{l}
\varepsilon \\
\chi
\end{array}\right)
$$

with: $\varepsilon(\mathrm{x})$, the axial strain ; $\chi(\mathrm{x})$, the curvature ; $\mathrm{N}$, normal force ; $\mathrm{M}$, bending moment ; $E_{p}$, elastic modulus at $P$.

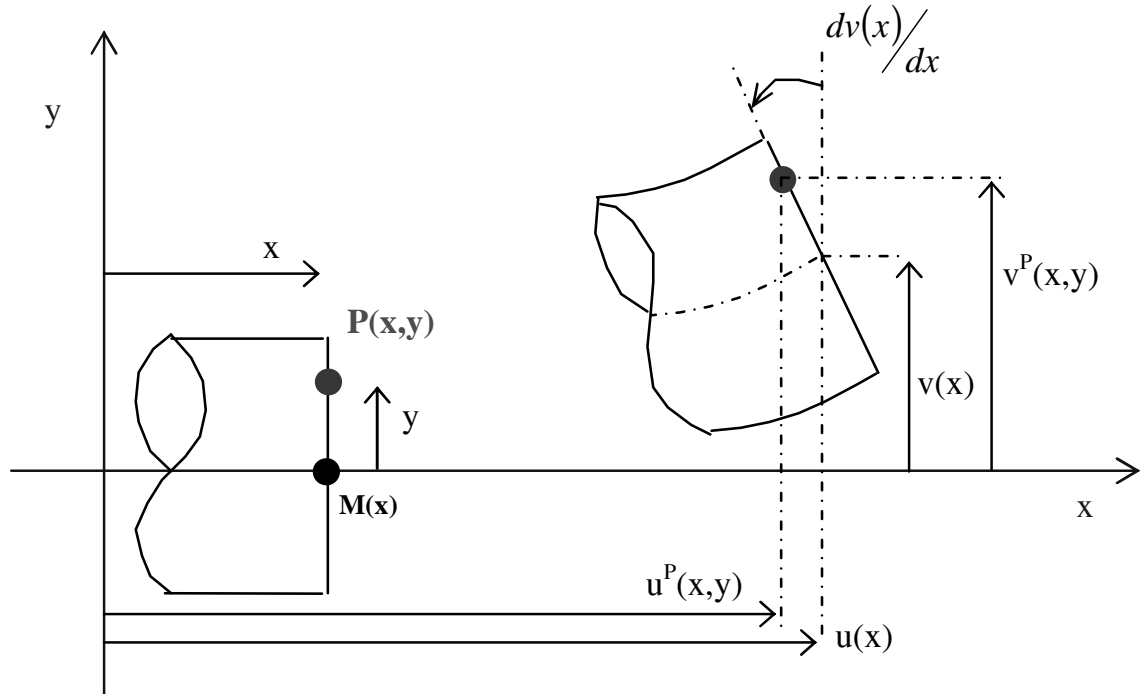

Figure 1. Section kinematics in Euler-Bernoulli beam

\subsection{Shape functions}

The shape functions usually used for Euler-Bernoulli beam elements are the Hermit polynomials (Hermite, 1873). The shape functions for axial displacement $\mathrm{u}(\mathrm{x})$ are linear $\left(\left(u_{1}, v_{1}, \theta_{1}, u_{2}, v_{2}, \theta_{2}\right)\right.$ nodes' displacements (Figure 2)):

$$
u(x)=N_{1}(x) \cdot u_{1}+N_{2}(x) \cdot u_{2}
$$

with $N_{1}(x)=1-\frac{x}{L} ; N_{2}(x)=\frac{x}{L}$

The shape functions for transverse displacement $\mathrm{v}(\mathrm{x})$ are cubic: 


$$
\begin{gathered}
v(x)=N_{3}(x) \cdot v_{1}+N_{4}(x) \cdot \theta_{1}+N_{5}(x) \cdot v_{2}+N_{6}(x) \cdot \theta_{2} \\
N_{3}(x)=1-3 \frac{x^{2}}{L^{2}}+2 \frac{x^{3}}{L^{3}} ; N_{4}(x)=\frac{x^{3}}{L^{2}}-2 \frac{x^{2}}{L}+x \\
N_{5}(x)=-2 \frac{x^{3}}{L^{3}}+3 \frac{x^{2}}{L^{2}} ; N_{6}(x)=\frac{x^{3}}{L^{2}}-\frac{x^{2}}{L}
\end{gathered}
$$

Figure 2. Nodes'displacements

And then, the displacements $(\mathrm{u}(\mathrm{x}), \mathrm{v}(\mathrm{x}))$ of any point $\mathrm{P}(\mathrm{x})$ from the beam's neutral axis are written as following :

$$
\begin{aligned}
& u(x)=\left(1-\frac{x}{L}\right) u_{1}+\left(\frac{x}{L}\right) u_{2} \\
& v(x)=\left(1-3 \frac{x^{2}}{L^{2}}+2 \frac{x^{3}}{L^{3}}\right) v_{1}+\left(\frac{x^{3}}{L^{2}}-2 \frac{x^{2}}{L}+x\right) \theta_{1} \\
& +\left(-2 \frac{x^{3}}{L^{3}}+3 \frac{x^{2}}{L^{2}}\right) v_{2}+\left(\frac{x^{3}}{L^{2}}-\frac{x^{2}}{L}\right) \theta_{2}
\end{aligned}
$$

So, whatever the loading, the deformation in the beam is given by the axial strain $\varepsilon(\mathrm{x})$ and by the curvature $\chi(\mathrm{x})$ :

$$
\begin{aligned}
& \varepsilon(x)=\frac{u_{2}-u_{1}}{L} \\
& \chi(x)=\left(-\frac{6}{L^{2}}+\frac{12 x}{L^{3}}\right) v_{1}+\left(\frac{6 x}{L^{2}}-\frac{4}{L}\right) \theta_{1}+\left(-\frac{12 x}{L^{3}}+\frac{6}{L^{2}}\right) v_{2} \\
& +\left(\frac{6 x}{L^{2}}-\frac{2}{L}\right) \theta_{2}
\end{aligned}
$$


The axial deformation is thus constant over the entire element length and the curvature varies linearly on the element.

\subsection{Need for enrichment}

A constant axial strain over the beam element's length can lead to various blockings. As soon as a variation of the axial strain due, either to loading (pile weight, dynamic loading), or to constitutive laws (plasticity, damage, non-linearity), or to the structure geometry, a blockade situation or an incompatibility is likely to appear and disturb the computational FEM process. Indeed, a nonlinear constitutive law or the beam cross-section's reference axis' choice (different from the neutral axis for example) can generate a non-controlled coupling between the normal force and the bending moment via $\left(-\int_{S} E_{P} y d S\right)$ in Equation [1] which links $(\mathrm{N}, \mathrm{M})$ and $(\varepsilon, \chi)$.

Moreover, it can be very important to obtain the same precision in the knowledge of the normal force and the bending moment on an element (Ragueneau et al., 2003). However, in the basic Euler-Bernoulli multifiber beam, an incompatibility in the writing of the axial strain exists because $\varepsilon(x)$ is constant [6] and $\chi(x)$ is linear [7]:

$$
\varepsilon_{\text {fibre }}=\varepsilon(x)-y_{\text {fibre }} \chi(x)
$$

To fix this problem, one solution consists in increasing the number of elements of the problem discretization. The precision of the axial strain description will depend on this number. Thus, this solution has important effects on the computing time and decreases the effectiveness of simplified models. Another solution, which will be developed in the following paragraph, is to change the description of the axial strain in the element by carrying out a kinematic enrichment.

\section{Enrichment}

The axial strain enrichment proposed uses the incompatible modes method, introduced in the 1970's by (Wilson et al., 1973) and then (Wilson et al., 1990). This method is presented and then the enrichment is carried out.

\subsection{Incompatible modes method}

The equilibrium equations, the kinematics equations and the constitutive laws are usually used to solve continuum mechanics problems. The incompatible modes method uses the Hu-Washizu three-field variational principle (Washizu, 1982) which 
consists in building the weak form of the preceding equations by introducing a virtual field of displacements, stresses and strains.

$u, \varepsilon, \sigma$ are respectively the fields of displacements, strains and stresses. For any virtual field, the following equations must be checked:

$$
\begin{aligned}
& \int_{\Omega} \hat{u}_{i, j} \cdot \sigma_{i j} d \Omega-W_{e x t}(\hat{u})=0 \\
& \int_{\Omega} \hat{\sigma}_{i j}\left(u_{i, j}-\varepsilon_{i j}\right) d \Omega=0 \\
& \int_{\Omega} \hat{\varepsilon}_{i j}\left(\sigma_{i j}-C_{i j k l} \varepsilon_{k l}\right) d \Omega=0
\end{aligned}
$$

with $W_{\text {ext }}(\hat{u})$, the external forces' work in the field of virtual displacements $\hat{u}$, and $C_{i j k l}$, constitutive law tensor.

A new decomposition of strains is then introduced, in which the added strains appear: $\tilde{\alpha}$ (real) and $\tilde{\tilde{\alpha}}$ (virtual):

$$
\begin{gathered}
\varepsilon_{i j}=u_{i, j}+\tilde{\alpha}_{i j} \\
\hat{\varepsilon}_{i j}=\hat{u}_{i, j}+\hat{\widetilde{\alpha}}_{i j}
\end{gathered}
$$

These expressions ([12] and [13]) are then introduced in relations [9], [10] and [11].

The incompatible modes method's idea is then to choose the stresses space, orthogonal with the added strains space. The equations to be solved become (Ibrahimbegovic, 2009):

$$
\begin{aligned}
& \int_{\Omega} \hat{u}_{i, j} C_{i j k l} \varepsilon_{k l} d \Omega-W_{e x t}(\hat{u})=0 \\
& \int_{\Omega} \hat{\tilde{\alpha}}_{i j} C_{i j k l} \varepsilon_{k l} d \Omega=0 \\
& \int_{\Omega} \hat{u}_{i, j}\left(\sigma_{i j}-C_{i j k l} \varepsilon_{k l}\right) d \Omega=0
\end{aligned}
$$

These expressions can be simplified by supposing that the strong form of the constitutive law is checked, namely: 


$$
\begin{aligned}
& \int_{\Omega} \hat{u}_{i, j} \sigma_{i j} d \Omega-W_{e x t}(\hat{u})=0 \\
& \int_{\Omega} \hat{\tilde{\alpha}}_{i j} \sigma_{i j} d \Omega=0 \\
& \sigma_{i j}=C_{i j k l} \varepsilon_{k l}
\end{aligned}
$$

This system must also be checked for a constant stress field, to guarantee the convergence of the problem. This condition is called "patch test".

\subsection{Formulation}

In order to fix the incompatibility on the integration described previously in 2.3, it is possible to carry out a kinematic enrichment of the element. In order to enrich the axial displacement, a new axial displacement, $\alpha$ and its shape function $\mathrm{N}_{0}(\mathrm{~s})$, corresponding to a new strain $\tilde{\alpha}$ called "enhanced strain” within the framework of the incompatible modes method, are introduced.

The axial displacement becomes:

$$
u(x)=N_{1}(x) \cdot u_{1}+N_{2}(x) \cdot u_{2}+N_{0}(x) \cdot \alpha
$$

and the axial strain is then:

$$
\varepsilon(x)=\frac{u_{2}-u_{1}}{L}+\tilde{\alpha}=\frac{u_{2}-u_{1}}{L}+\frac{d N_{0}(x)}{d x} \alpha
$$

In order to have the axial strain and the curvature described with a linear function of $\mathrm{x}, \mathrm{N}_{0}(\mathrm{x})$ must be a quadratic function of $\mathrm{x}$. Moreover, $\mathrm{N}_{0}(\mathrm{x})$ should not change the values of $u(x)$ in $x=0$ and $x=L$. The function chosen for $N_{0}(x)$ is a "bubble" function (Figure 3):

$$
N_{0}(x)=-4 \frac{x^{2}}{L^{2}}+4 \frac{x}{L}
$$

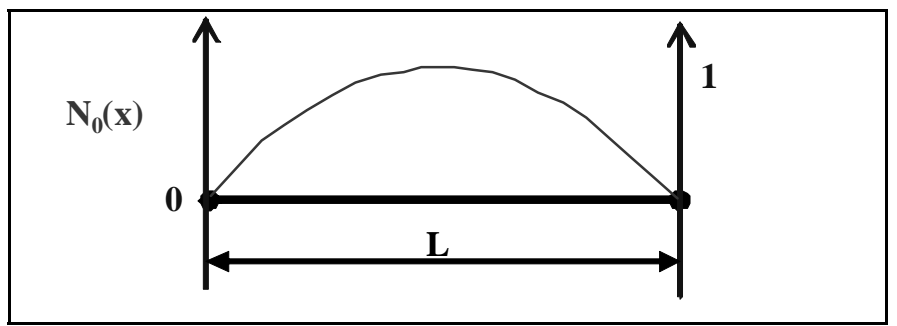

Figure 3. «Bubble» shape function for added displacement 
It comes:

$$
\begin{aligned}
& u(x)=\left(1-\frac{x}{L}\right) u_{1}+\frac{x}{L} u_{2}+\left(-4 \frac{x^{2}}{L^{2}}+4 \frac{x}{L}\right) \alpha \\
& v(x)=\left(1-3 \frac{x^{2}}{L^{2}}+2 \frac{x^{3}}{L^{3}}\right) v_{1}+\left(\frac{x^{3}}{L^{2}}-2 \frac{x^{2}}{L}+x\right) \theta_{1} \\
& +\left(-2 \frac{x^{3}}{L^{3}}+3 \frac{x^{2}}{L^{2}}\right) v_{2}+\left(\frac{x^{3}}{L^{2}}-\frac{x^{2}}{L}\right) \theta_{2}
\end{aligned}
$$

And then:

$$
\begin{aligned}
& \varepsilon(x)=\frac{u_{2}-u_{1}}{L}+\left(-\frac{8 x}{L^{2}}+\frac{4}{L}\right) \alpha \\
& \chi(x)=\left(-\frac{6}{L^{2}}+\frac{12 x}{L^{3}}\right) v_{1}+\left(\frac{6 x}{L^{2}}-\frac{4}{L}\right) \theta_{1}+\left(-\frac{12 x}{L^{3}}+\frac{6}{L^{2}}\right) v_{2} \\
& +\left(\frac{6 x}{L^{2}}-\frac{2}{L}\right) \theta_{2}
\end{aligned}
$$

In this way, there is no incompatibility between the axial strain's interpolation and the curvature's one: both are linear functions of x-coordinate. The incompatible modes method will enable to calculate the added strain and the added displacement, for enriching the axial strain's description of the Euler-Bernoulli multifiber beam element.

\section{Numerical implementation}

The enrichment has been established in the FEM code FEAP (Finite Element Analysis Program) (Taylor, 1996). Indeed, there is in this code a multifiber beam elements library, FEDEAS (Filippou, 1996), with a Euler-Bernoulli multifiber beam element. Moreover, the number of nodal displacements is reduced by removing the ones which create only rigid body motions. Instead of as the beam nodes' displacements $U=\left(u_{1}, v_{1}, \theta_{1}, u_{2}, v_{2}, \theta_{2}\right), V$ is used:

$$
V=\left[u_{2}-u_{1} ; \theta_{1}-\frac{v_{2}-v_{1}}{L} ; \theta_{2}-\frac{v_{2}-v_{1}}{L}\right]=\left[u_{2}-u_{1} ; \Theta_{1} ; \Theta_{2}\right]
$$


This allows reducing computation time considerably, without harming the results' quality since this simplification is done without loss of information (Filippou, 1996).

Thus, the unknown factors of the Euler-Bernoulli beam element are:

$V=\left[\begin{array}{lll}u_{2}-u_{1} & \Theta_{1} & \Theta_{2}\end{array}\right]$ : nodes' displacements

$V_{S}=\left[\begin{array}{ll}\varepsilon_{S} & \chi_{S}\end{array}\right]:$ strain and curvature in cross-section

$F_{S}=\left[\begin{array}{ll}N_{S} & M_{S}\end{array}\right]:$ forces in cross section

$\hat{V}, \hat{V}_{S}, \hat{F}_{S}$ respective virtual fields

For a cross-section S, located at x-coordinate, one can write:

$$
V_{s}(x)=B V+\tilde{\alpha}=B V+G \alpha
$$

with:

- the derivative of the shape functions associated with $V$ :

$$
B=\left[\begin{array}{ccc}
\frac{1}{L} & 0 & 0 \\
0 & \frac{-4}{L}+\frac{6 x}{L^{2}} & \frac{-2}{L}+\frac{6 x}{L^{2}}
\end{array}\right]
$$

- the derivative of the shape function associated with $\alpha$, the added displacement :

$$
G=\left[\begin{array}{c}
\frac{d N_{0}}{d x} \\
0
\end{array}\right]=\left[\begin{array}{c}
\frac{-8 x}{L^{2}}+\frac{4}{L} \\
0
\end{array}\right]
$$

For $x \in[0 ; L]$, this leads to:

$$
\begin{aligned}
& {\left[\begin{array}{c}
\varepsilon_{S} \\
\chi_{S}
\end{array}\right]=\left[\begin{array}{ccc}
\frac{1}{L} & 0 & 0 \\
0 & \frac{-4}{L}+\frac{6 x}{L^{2}} & \frac{-2}{L}+\frac{6 x}{L^{2}}
\end{array}\right]\left[\begin{array}{c}
u 2-u 1 \\
\Theta_{1} \\
\Theta_{2}
\end{array}\right]} \\
& +\left[\begin{array}{c}
\frac{-8 x}{L^{2}}+\frac{4}{L} \\
0
\end{array}\right] \alpha
\end{aligned}
$$


These expressions are replaced in the equations of the incompatible modes method's final system [17] [18]:

$$
\begin{aligned}
& \int_{\Omega} B \cdot \hat{V} \cdot F_{S} d \Omega-W_{\text {ext }}(\hat{V})=0 \\
& \int_{\text {élément }} G \cdot \hat{\alpha} \cdot F_{S} d \Omega_{e}=0
\end{aligned}
$$

The system becomes:

$$
\underset{e=1}{e=N_{\text {elem }}}\left(r^{e}-f^{e}\right)=0
$$

Global equation to check over the modeled structure

$$
h^{e}=0, \forall e \in\left[1, N_{\text {elem }}\right]
$$

Local equation to check over every element

with:

$r^{e}=\int_{\text {élément }} B^{T} \cdot F_{S} d \Omega_{e}:$ internal forces; $f_{e}:$ external forces;

$h_{e}=\int_{\text {élément }} G^{T} \cdot \vec{F}_{S} d \Omega_{e}$

The new element has to check the "patch test”, namely:

$$
\int_{\text {élément }} G^{T} d \Omega_{e}=0
$$

However, $G=\left[\begin{array}{c}\frac{d N}{d x} \\ 0\end{array}\right]=\left[\begin{array}{c}\frac{-8 x}{L^{2}}+\frac{4}{L} \\ 0\end{array}\right]$, from where:

$\int_{0}^{L}\left(\frac{-8 x}{L^{2}}+\frac{4}{L}\right) d x=\left[\frac{-4 x^{2}}{L^{2}}+\frac{4 x}{L}\right]_{0}^{L}=0$

Thus, function G checks the "patch test” well.

The system is solved with a sequential method. The variable for global computation index is called i. For each i iteration, the complete system can be written as:

$$
\left[\begin{array}{ll}
K^{e} & F^{e} \\
F^{e} & H^{e}
\end{array}\right]\left[\begin{array}{l}
\Delta U^{e} \\
\Delta \alpha^{e}
\end{array}\right]=\left[\begin{array}{c}
f^{e}-r^{e} \\
-h^{e}
\end{array}\right]
$$


with: $F^{e}=\int_{\text {élément }} B^{T} \cdot C_{S} \cdot G \cdot d \Omega_{e} ; \quad H^{e}=\int_{\text {élément }} G^{T} \cdot C_{S} \cdot G \cdot d \Omega_{e} ; \Delta U^{e}$, the displacements' increment; $K^{e}=\int_{\text {élément }} B^{T} \cdot C_{S} \cdot B \cdot d \Omega_{e}$, the element's rigidity matrix ; $\Delta \alpha^{e}$, incompatible mode's increment

The displacement's value of incompatible mode being independently defined in each element, one can carry out a static condensation of this system, in order to have only the global equation to solve (the calculation of $\alpha$ is realized at the level of the element).

One obtains then:

$$
\hat{K}^{e} \cdot \Delta U^{e}=\hat{f}^{e} \text {, with } \hat{K}^{e}=K^{e}-F^{e} \cdot\left(H^{e}\right)^{-1} \cdot\left(F^{e}\right)^{T}
$$

The computation of $\alpha$ is solved by using an iterative method, at the level of the element. By noting $i$, the index of "global" iteration, and $j$, the index of local iteration:

$$
\alpha_{(i)}^{(j+1)}=\alpha_{(i)}^{(j)}-H_{(i)}^{e(j)-1} h_{(i)}^{e(j)}
$$

With: $H^{e(i)}=\int_{0}^{L} G^{T} C_{s} G d x, C_{s}=\left[\begin{array}{cc}\int_{\text {section }} E_{i} A_{i} & -\int_{\text {section }} E_{i} A_{i} y_{i} \\ -\int_{\text {section }} E_{i} A_{i} y_{i} & \int_{\text {section }} E_{i} A_{i} y_{i}^{2}\end{array}\right]$ and $h^{e(i)}=\int_{0}^{L} G^{T}\left[\begin{array}{l}N \\ M\end{array}\right] d x$

The converged value of $\alpha$ parameter is obtained when:

$$
h^{e}\left(U^{(i)}, \alpha^{(i)}\right)=0
$$

\section{Academic test}

\subsection{Test description}

In order to highlight the enrichment, the following test is proposed. The structure tested is a beam with rectangular constant cross-section S and length $\mathrm{L}$. It is on two supports: pivot at the left end of the beam and simple support at the right end. Firstly, an increasing axial load $\mathrm{F}$ is applied at the right end node of the beam, up to a $F_{\max }$ value. Then, $F$ value is maintained constant and equal to $F_{\max }$, and rotation $\theta$ of the left end node of the beam is increased or decreased linearly. 
If the beam's material evolves with a non-elastic law, then the axial strain will not stay constant. Indeed, it will be larger in the already plasticized or damaged zones, because of the shift of the neutral axis. Thus, a lot of basic Bernoulli beam elements are needed to model this behavior.

Only two new enriched elements are enough to reproduce the evolution of the axial strain along the beam (one cannot go down below two elements to discretize a structure with FEAP, because of the code operation).

The geometrical characteristics of the tested beam are: $\mathrm{L}=2 \mathrm{~m}$ and $\mathrm{S}=0,2 \mathrm{~m} \times 0,2 \mathrm{~m}$

The beam's material is an elasto-plastic steel with work hardening (Young modulus $=200000 \mathrm{MPa}$, elastic limit $=500 \mathrm{MPa}$, work hardening $=1 \%)$. The beam is modeled with two multifiber beam elements (Figure 4). Each cross-section is made of $(3 \times 3)$ fibers. The axial load $F_{\max }$ is $19 \mathrm{MN}$. The $\theta$ rotation is increased up to 10.E-3 then decreased to 5.E-3 then increased again, up to 20.E-3.

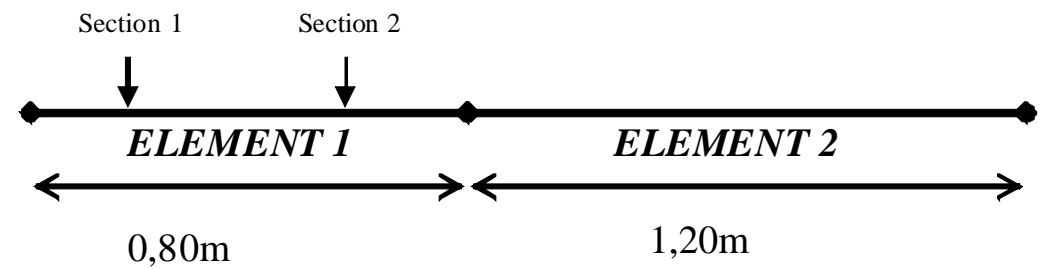

Figure 4. Beam modeling

\subsection{Results}

The same computation is carried out with the basic elements and with the enriched ones.

With the basic elements, the axial strains of Section 1 and Section 2 are identical (that is normal for this kind of element). As soon as some fibers start to plasticize (with the increase of the applied rotation), the element does not manage to preserve a constant normal force in its two sections, to ensure global equilibrium. There is also a very bad convergence, even a divergence at the global level.

The results provided by the enriched multifiber beam element seem very satisfactory. Indeed, the evolution of the axial strain of Section 1 and that of Section 2 are different (Figure 5) within element 1, as soon as some fibers start to plasticize (Figure 6). This means that the incompatible mode parameter, $\alpha$, that was added, functions correctly and makes it possible to have a non constant axial strain over the element. The element manages to preserve a constant normal force in both sections (Figure 7). Moreover, a very good convergence is observed. A synthesis of 
the evolution of strains and stresses in Sections 1 and 2 of element 1 during the test (during the increase in imposed rotation) is proposed in Table 1.

This test has been carried out with other nonlinear constitutive laws or with composite sections and has also given very good performances (Casaux, 2003).

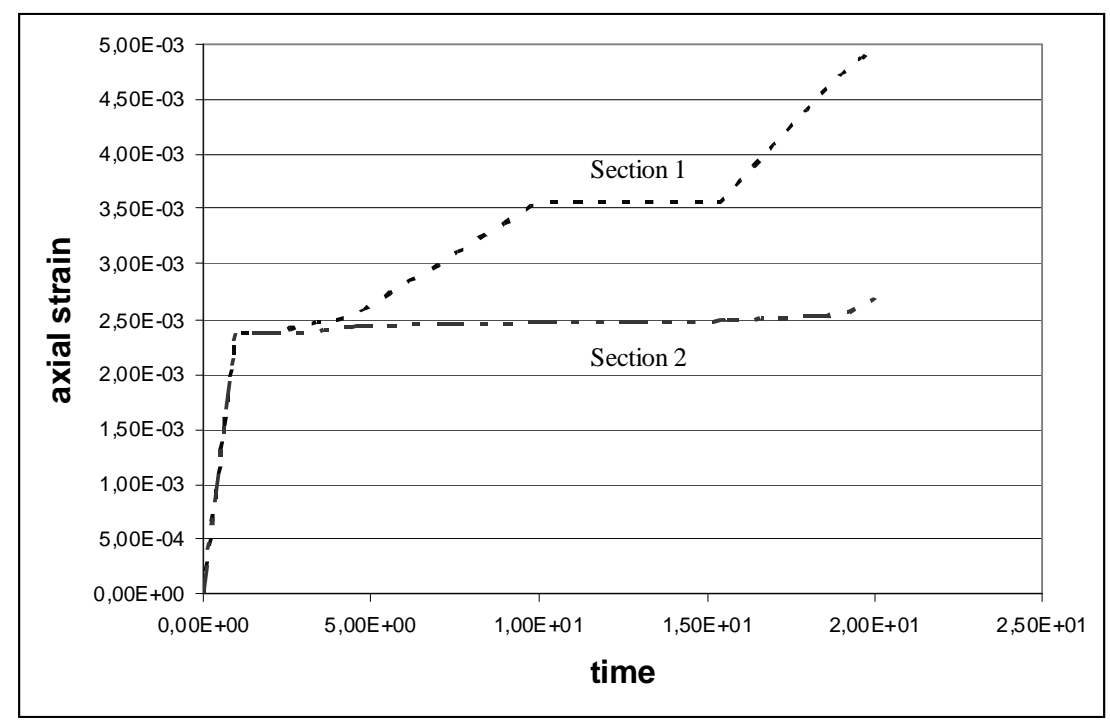

Figure 5. Sections' axial strains in element 1 with enriched elements
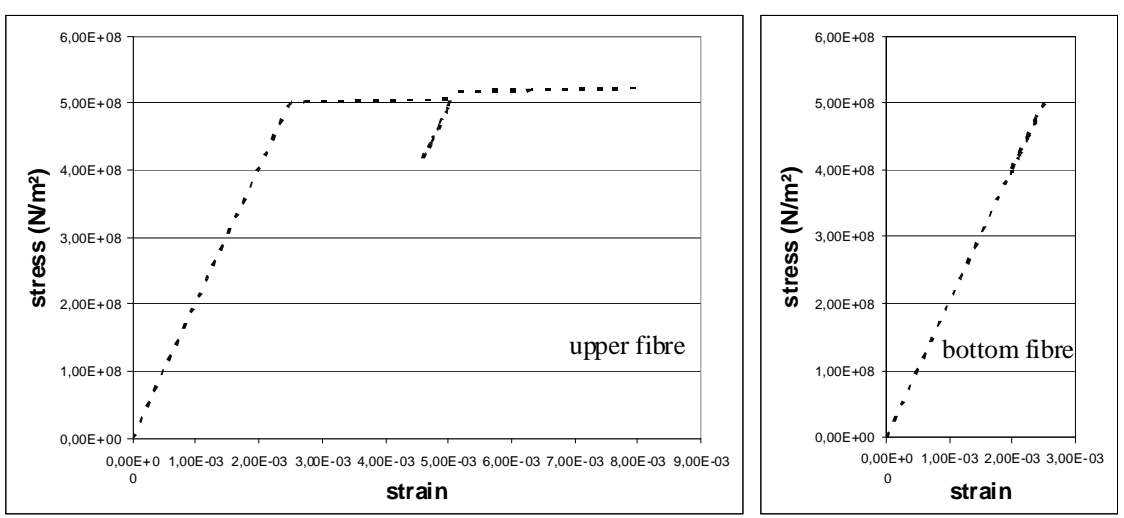

Figure 6. Stress/strain evolutions for fibres in [section 1 -element 1] during the test 
Table 1. Strain-stress evolution in element 1 during the test for basic or enriched elements
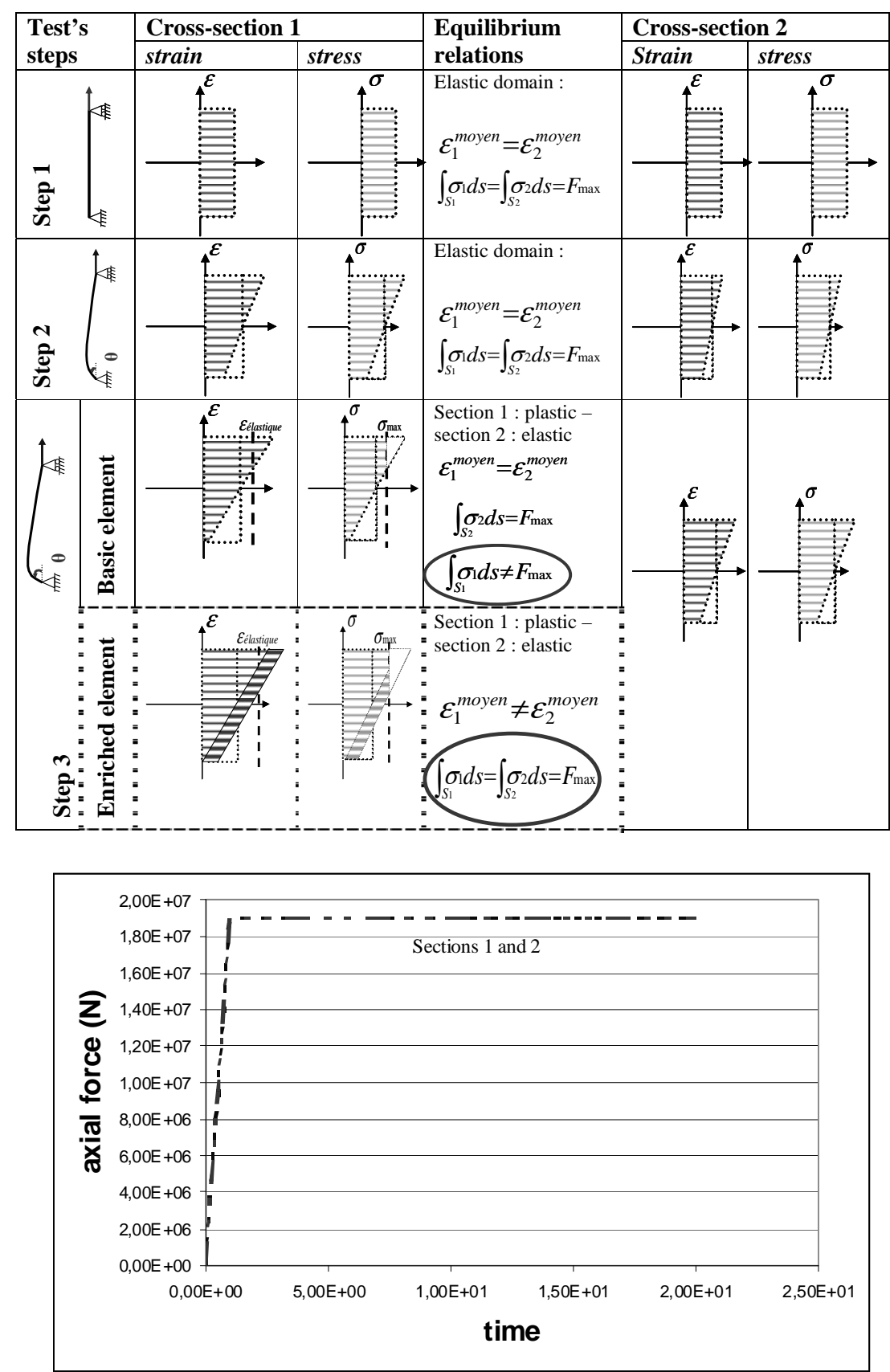

Figure 7. Normal forces in element 1 with enriched elements 


\section{Conclusion}

After detailing the Euler-Bernoulli multifiber beam element's characteristics and measuring its advantages and disadvantages, an incompatibility in the description of the axial strain has been highlighted. This incompatibility can prevent the element from describing correctly the behavior of structures subjected to coupled loadings. An improvement, based on the incompatible modes method, has been proposed in order to cure this incompatibility. The new enriched multifiber beam element has a linear variation of its curvature according to its length, that gives him more flexible kinematics. The beam's strain allows a controlled coupling between normal force and bending moment. The academic test has shown the efficiency of this new element compared to the basic Euler-Bernoulli one. Since this incompatibility has been corrected, others enrichments (Mazars et al., 2006) can be coupled to this one, and keep on improving multifiber beam modeling.

\section{References}

Casaux G., Modélisation tridimensionnelle du comportement sismique d’ouvrages en béton armé - Développement de méthodes simplifiées, Ph.D.thesis, LMT - Ecole Normale Supérieure de Cachan, 2003.

Filippou F.C., "Non linear static and dynamic analysis for evaluation of structures”, $3^{\text {rd }}$ European Conference on Structural Dynamics Eurodyn, Florence, Italy, 1996, p. 395402.

Hermite C., Sur la fonction exponentielle, Comptes rendus de l'Académie des Sciences, tome 77, Bibliothèque Nationale de France, 1873.

Ibrahimbegovic A., Wilson E.L., “A modified method of incompatible modes”, Commun. Appl. Numer. methods, 7, 1991, p. 187-194.

Ibrahimbegovic A., Nonlinear Solid Mechanics: Theoretical Formulations and Finite Element Solution Methods, Springer, 2009.

Mazars J., Kotronis P., Ragueneau R.,.Casaux G., U”sing multifiber beams to account for shear and torsion - Applications to concrete structural elements”, Comput. Methods Appl. Mech. Engrg. 195, 2006, p. 7264-7281.

Ragueneau F., Casaux G., Mazars J., « CAMUS 2000 benchmark analyses through multifiber beam formulation coupled to continuum damage mechanics ", Proceedings $6^{e}$ Colloque National de l'Association Française du Génie Parasismique, AFPS, Ecole Polytechnique, Palaiseau, 2003.

Spacone E., Ciampi V., Filippou F., “Mixed formulation of non-linear beam finite element”, Comp. Struct., vol. 58, $\mathrm{n}^{\circ}$ 1,1996, p. 71-83.

Taylor R.L., FEAP: a finite element analysis program, University of California, Berkeley, 1996.

Washizu K., Variational Methods in Elasticity and Plasticity, Pergamon Press, 1982. 
Wilson E.L., Taylor R.L., Doherty W.P. and Ghaboussi J., "Incompatible displacement models”, S.J. Fenves et al. (eds.), Numerical and Computer Methods in Structural Mechanics, Academic Press, New York, 1973, p. 43-57.

Wilson E.L., Ibrahimbegovic A., "Use of incompatible displacement modes for the calculation of element stiffness or stresses”, Finite elements in analysis and design,vol. 7, 1990, p. 229-241.

Received: 2 February 2010

Accepted: 5 January 2012 